Brazilian Journal

of Chemical

ISSN 0104-6632

Printed in Brazil

Engineering

\title{
LIQUID-LIQUID EQUILIBRIA DATA OF N-METHYL-2-HYDROXYETHYL AMMONIUM ALKYLATES WITH BUTANOL+WATER AND PENTANOL+WATER AT 293.15K, 313.15K AND $333.15 \mathrm{~K}$
}

\author{
Karina S. Amorim ${ }^{1}$, Amanda G. Soares ${ }^{1}$, Fernanda M. Ganem ${ }^{1}$, Erik A. \\ de Souza ${ }^{1}$ and Silvana Mattedi ${ }^{1, *}$
}

\author{
${ }^{1}$ Chemical Engineering Graduate Program, Polytechnic School - Federal University of Bahia, \\ Rua Aristides Novis, 2 Federação, CEP: 40.210-630 .Salvador - BA, Brazil.
}

(Submitted: May 1, 2016; Revised: November 4, 2016; Accepted: November 29, 2016)

\begin{abstract}
Ionic liquids (ILs) are organic salts with low volatility and have different applications including organic synthesis, separation and extraction processes, fuel cell electrolyte, surfactants, among others. Protic ILs based on the N-methyl-2-hydroxyethyl ammonium cation and short chain carboxylic anions have simple synthesis and purification. However, as new substances few data are available for their thermodynamic properties and phase equilibria. In order to investigate the partition of these substances in alcohol-water, liquid-liquid equilibria data were determined experimentally for three ionic liquids (formed by the cation N-methyl-2-hydroxyethyl ammonium (m-2HEA) and the anions propionate (Pr), butanoate (B) and pentanoate (P)) with butanol and pentanol, at three different temperatures: $293.15 \mathrm{~K}, 313.15 \mathrm{~K}$ and $333.15 \mathrm{~K}$. The binodal curves are obtained by the turbidity method with gravimetric determination of all the components, curves are fitted using the Merchuk method. The tie-lines are also gravimetrically determined. The results confirm the high hydrophilicity of these ionic liquids, with partition coefficients of water-butanol and water-pentanol lower than 1. Data were correlated with UNIFAC.
\end{abstract}

Keywords: Ionic liquid, Partition, Solubility, Thermodynamic equilibrium.

\section{INTRODUCTION}

Ionic liquids are a class of organic or mixed salts that have a melting point lower than $373.15 \mathrm{~K}$. These liquids have many applications in the fields of electrochemistry, catalysis, separations, surfactants, electrolytes for fuel cells, among others. They show great advantage compared to traditional organic solvents due to their extremely low volatility that prevents air contamination. Because of this feature, many works in the past claimed that they were green solvents, but many ionic liquids are toxic (Latala et al., 2005) and effluents or spills could contaminate aquifers and consequently affect the flora and the fauna present in the

* Corresponding author: E-mail: silvana@ufba.br 
enviroment. Studies indicate that ionic liquids based on imidazolium are more toxic than protic ionic liquids (PIL) based on amines and short - chain acids and that the toxicity is related to the hydrophilicity / lipophilicity of the ionic liquid (Peric et al., 2014, Oliveira et al. 2016). They are formed by a Bronsted- Lowry acid-base reaction (Drummond and Kennedy, 2009) that, unlike aprotic ionic liquids, have labile protons and are able to form a hydrogen bond network. Furthermore, LIPs have a low preparation cost because of a simple one-step synthesis (Alvarez et al., 2010), not requiring additional purification steps. N-Methyl2-hydroxyethylammonium carboxylates could be used to separate undesirable sulphur components from diesel (Oliveira et al., 2012), are selective to carbon dioxide/methane separation (Carvalho and Coutinho, 2011) and could modify silica to encapsulate enzymes (Souza et al., 2013, Oliveira et al., 2014). The development of processes based on these compounds needs a lot of data and models that represent these data. Thermodynamic properties (density at different pressures, pure and in mixtures, sound velocities, calorific capacities, viscosities and refractive index) of these compounds have been studied (Alvarez et al, 2010; Alvarez et al., 2013; Talavera-Prieto et al., 2014; Li et al., 2015; Li et al. 2016); however, few liquid-liquid data are available in the literature. Water and alcohol form a two phase system which is interesting to study as it could be used to purify substances. The aim of this work is to obtain liquid-liquid equilibrium data in two different systems: butanol + ionic liquid + water and pentanol + ionic liquid + water.

\section{EXPERIMENTAL}

The properties of each compound are shown in Table 1 . The ionic liquids used are simply synthesized in the laboratory from short - chain carboxylic acids and substituted amines with 1: 1 stoichiometry. The amine is placed in a three neck flask equipped with a reflux condenser. The acid is added dropwise to the flask at $25^{\circ} \mathrm{C}$ under stirring by magnetic stirrer bar. The synthesis is carried out in an inert atmosphere using nitrogen gas avoiding parallel reactions. In this work we used N-methyl-2hydroxyethylammonium propionate (m-2HEAPr) $\mathrm{N}$-methyl-2-hydroxyethylammonium butanoate (m-2HEAB) and N-methyl-2-hydroxyethylamonium pentanoate (m-2HEAP). The synthesis was performed in a nitrogen atmosphere and at the end of the reaction nitrogen was bubbled for a half hour to remove unreacted compounds. The reaction is a Bronsted acid-base neutralization forming a protic ionic liquid. The water content was measured using a Mettler Toledo V20 volumetric Karl Fischer titrator before each experiment and considered in the calculation. The ${ }^{1} \mathrm{H}-\mathrm{NMR}$ spectra of the ionic liquids were obtained to confirm the structure and purity of the synthesized compounds.

Table 1. Properties of the pure components.

\begin{tabular}{lcccc}
\hline \multicolumn{1}{c}{ Component } & $\begin{array}{c}\text { Molar Mass } \\
\left({\left.\mathrm{g} . \mathrm{mol}^{-1}\right)}^{-1}\right.\end{array}$ & Purity (g/g) & Determined by & Supplier \\
\hline 2-hydroxyethylamine & 61.08 & 0.99 & $\mathrm{CG}^{1}$ & Sigma-Aldrich \\
Propionic acid & 74.09 & 0.98 & $\mathrm{CG}^{1}$ & Merck \\
Butanoic acid & 88,11 & 0.98 & $\mathrm{CG}^{1}$ & Merck \\
Pentanoic acid & 102.13 & 0.98 & $\mathrm{CG}^{1}$ & Merck \\
N-methyl-2-hydroxyethylamine & 73.14 & 0.987 & $\mathrm{CG}^{1}$ & Synth \\
m-2HEAPr & 149.19 & $>0.99$ & $\mathrm{NMR}^{2}$ & Synthesized for this work \\
m-2HEAB & 163.22 & $>0.99$ & $\mathrm{NMR}^{2}$ & Synthesized for this work \\
m-2HEAP & 177.24 & $>0.99$ & $\mathrm{NMR}^{2}$ & Synthesized for this work \\
1-Pentanol & 88,15 & 0.99 & $\mathrm{CG}^{3}$ & Sigma-Aldrich \\
1-Butanol & 74.12 & 0.99 & $\mathrm{CG}^{3}$ & Sigma-Aldrich \\
Water & 18.0 & $>0.99$ & Condutivimetry & MilliQ and double distilled \\
\hline
\end{tabular}

${ }^{1} \mathrm{GC}$ performed by the manufacturer and used without further purification. ${ }^{2}$ Verified through NMR spectra, water content measured by Karl-Fisher titration before each experiment and considered to calculate compositions. ${ }^{3}$ Confirmed by GC for this work.

Solubility data for the ternary mixture were determined using the cloud point method (Nemati-Kande et al., 2012). The vial was closed with a septum and a cap to prevent any evaporation and kept stirring using a magnetic bar. Each component was added to the vial using a syringe.

The temperature of the system was controlled by a water jacket with an uncertainty of $\pm 0.1 \mathrm{~K}$. Mass 
changes were measured with an analytical balance (Shimadzu AX200, accuracy of $\pm 0.0001 \mathrm{~g}$ ) and used to calculate the composition of components in the binodal curve. The values of the compositions of ILs were corrected according to water content of each compound. The experimental binodal curves were fitted using Merchuk et al. (1998) (equation 1),

$$
100 w_{a}=\operatorname{Aexp}\left[\left(B .100 w_{\text {water }}^{0.5}\right)-\left(C .100 w_{\text {water }}^{3}\right)\right]
$$

where $100 w_{a}$ and $100 w_{\text {water }}$ are the alcohol and water weight fractions, respectively, and $\mathrm{A}, \mathrm{B}$, and $\mathrm{C}$ are fitted parameters obtained by least-squares regression.

Mixtures with compositions in the region of immiscibility were prepared by weighing known masses of each component and discharged in a glass cell with a stopper. The prepared mixtures were placed in a thermostatic bath and stirred for 40 minutes. Afterward, the systems were centrifuged for 10 minutes to facilitate the separation and then allowed to rest for 24 hours at controlled temperature to guarantee the equilibrium. Afterwards, the organic phase was carefully collected and weighed.

Data were modelled with UNIFAC. The parameter estimation $a_{m n}$ from the UNIFAC model was done by minimizing the objective function (eq. 2).

$$
F_{o b j}=\sum_{k}^{D} \sum_{j}^{N_{k}} \sum_{i}^{C_{k}-1}\left[\left(x_{i j k}^{\alpha}-x_{i j k}^{\alpha, e s t i m .}\right)^{2}+\left(x_{i j k}^{\beta}-x_{i j k}^{\beta, e s t i m . ~}\right)^{2}\right]
$$

D represents the quantity of systems used, $N_{k}$ and $C_{k}$ the number of experimental points and components, respectively, in each system $\mathrm{k}$. The TML-LLE program (Stragevitch and d'Ávila, 1997), based on a modified simplex procedure, was used to estimate the interaction parameters. The code could treat several systems with different numbers of components and at several temperatures simultaneously (Santos, 2005). Each ionic liquid (cation-anion pair) was considered as a new single group and only interaction parameters between them and the solvent groups need to be reestimated.

Volume and area (R and Q) parameters for ionic liquid groups are not available in the literature and were derived by optimizing the ionic liquid geometry with density-functional theory (DFT) performed with a quantum mechanics software and then calculating van der Waals area and volume with the polarizable continuum model (PCM).

\section{RESULTS}

The binodal curves for each water + IL + butanol systems and water + IL + pentanol system at three different temperatures and at atmospheric pressure
$(101.2 \mathrm{kPa})$ can be seen in Figures 1 - 6. It is important to note that the room pressure was checked every day during the experiments, and an average pressure of $101.2 \mathrm{kPa}$ was obtained with standard deviation of $0.1 \mathrm{kPa}$. The experimental binodal data of all systems studied are shown in Tables $2-7$. The uncertainties in the values of the mass fractions of each system component can be determined by calculating the uncertainty of adding an extra drop of water or alcohol to the system during the determination of the binodal through the cloud point method. The mass and its uncertainty for an extra drop in the aqueous phase was $0.0151 \pm$ $0.00003 \mathrm{~g}$, for the butanol phase was $0.0052 \pm 0.0002 \mathrm{~g}$ and for the pentanol phase was $0.0063 \pm 0.00004 \mathrm{~g}$. These values were used to calculate the combined uncertainty in each phase. It was observed that the imiscibility gap decreases with increasing temperature. This behavior is expected as it is similar to other water-alcohol systems, the salting-in effect of the IL increasing with temperature. Similar behavior was observed by Nann et al. (2013) with aprotic ionic liquids. By analyzing IL behavior at the same temperature, it is observed that the increase in the anion chain length induces an increase in solubility, decreasing the miscibility gap. The hydrophobicity of the ionic liquid is increased by the anion chain and it helps to enhance the alcohol-water mutual solubility as can be seen in Figs . 7 - 12. Thus, the IL surfactant effect is enhanced as the anion chain length is increased, which is also an expected behavior (Santos et al., 2010; Greaves and Drummond, 2014).

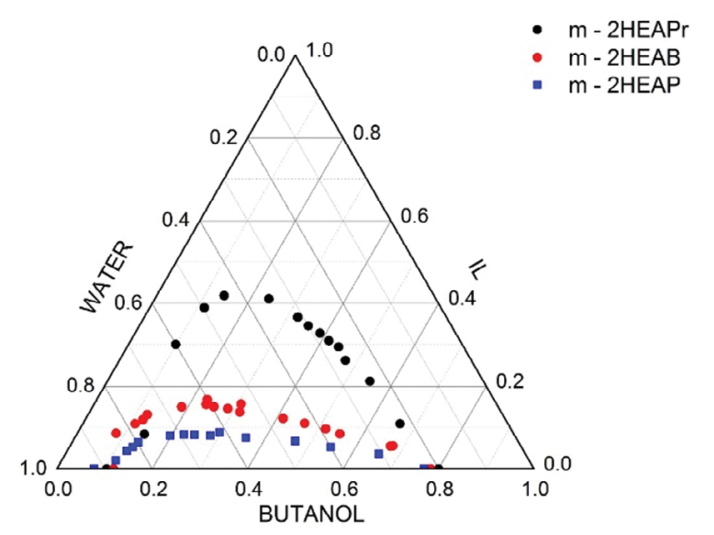

Figure 1: Binodal curves for water $+\mathrm{IL}+$ butanol, in mass fraction, at 293.15K: m-2HEPr (black), m-2HEAB (red) and m-2HEAP (blue). 


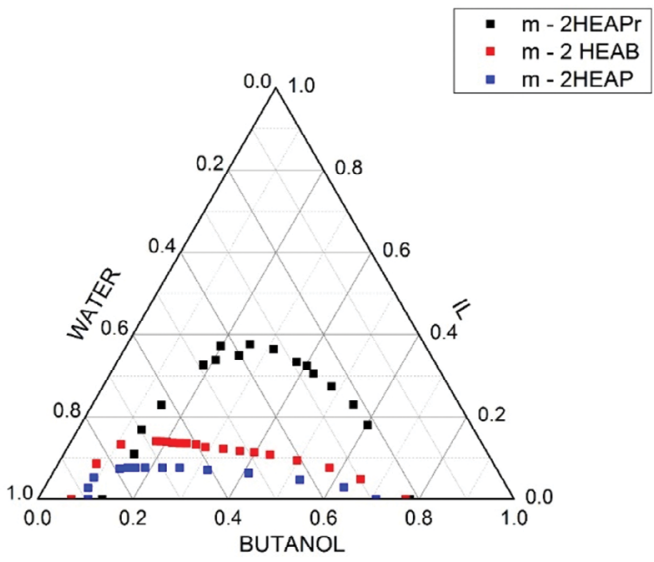

Figure 2: Binodal curves for water $+\mathrm{IL}+$ butanol, in mass fraction, at 313.15K: m-2HEPr (black), m-2HEAB (red) and m-2HEAP (blue).

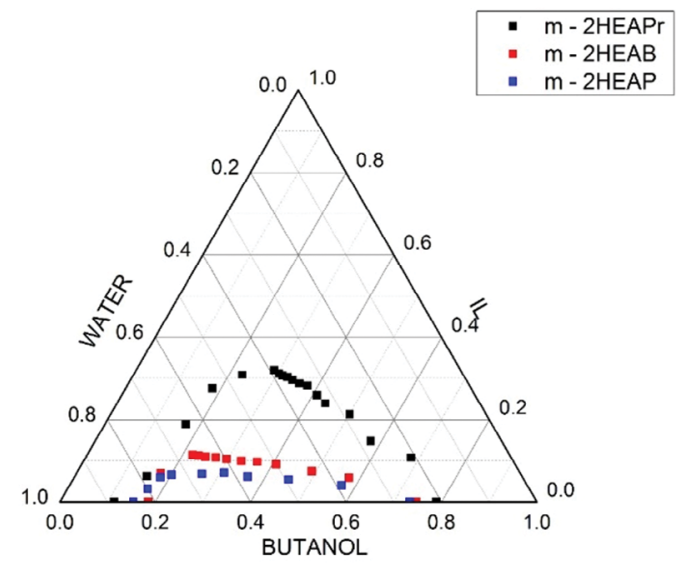

Figure 3: Binodal curves for water $+\mathrm{IL}+$ butanol, in mass fraction, at $333.15 \mathrm{~K}: \mathrm{m}-2 \mathrm{HEPr}$ (black), m-2HEAB (red) and m-2HEAP (blue).

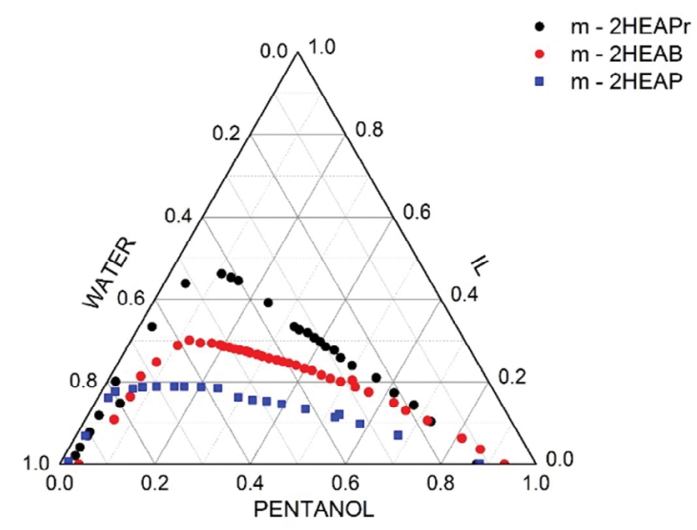

Figure 4: Binodal curves for water + IL + pentanol, in mass fraction, at 293.15K: m-2HEPr (black), m-2HEAB (red) and m-2HEAP (blue).

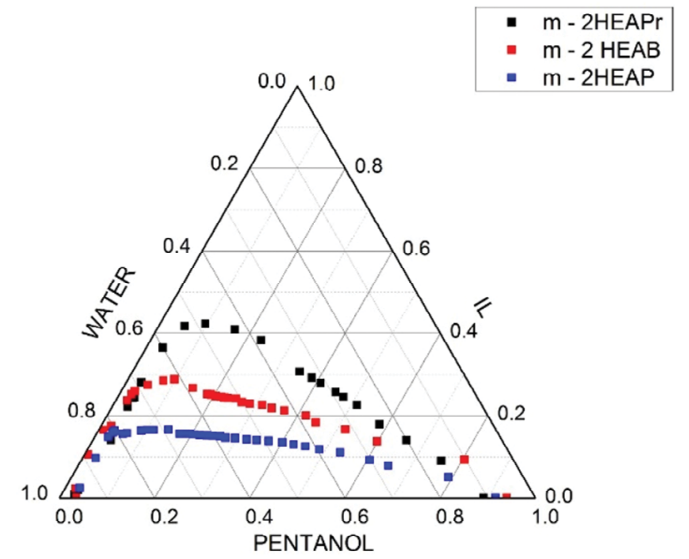

Figure 5: Binodal curves for water + IL + pentanol, in mass fraction, at $313.15 \mathrm{~K}: \mathrm{m}-2 \mathrm{HEPr}$ (black), m-2HEAB (red) and m-2HEAP (blue).

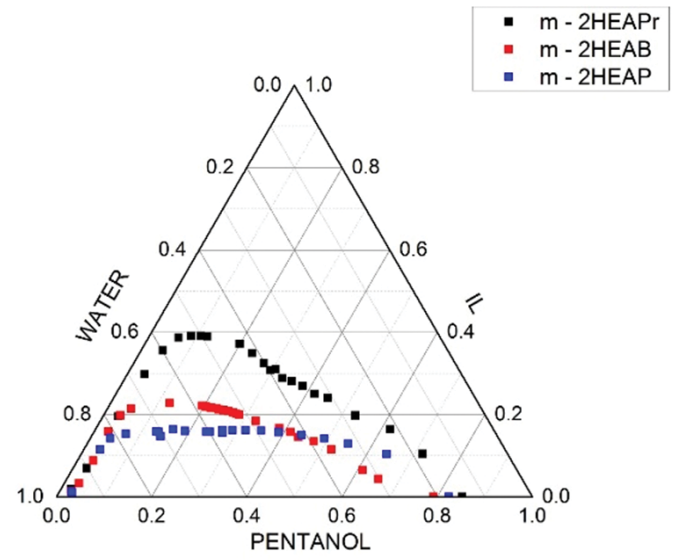

Figure 6: Binodal curves for water + IL + pentanol, in mass fraction, at 333.15K: m-2HEPr (black), m-2HEAB (red) and m-2HEAP (blue). 
Table 2. Binodal curve data: Mass fraction (w) of Cloud Points of water (1) + m-2HEAPr (2) + Butanol (3) Systems at $\mathrm{P}=101.2 \mathrm{kPa}$ and $293.15,313.15$ and $333.15 \mathrm{~K}$

\begin{tabular}{ccccccccc}
\hline & $\mathbf{2 9 3 . 1 5 K}$ & & & $\mathbf{3 1 3 . 1 5 K}$ & & \multicolumn{3}{c}{$\mathbf{3 3 3 . 1 5}$} \\
\hline $\mathbf{w}_{\mathbf{1}}$ & $\mathbf{w}_{\mathbf{2}}$ & $\mathbf{w}_{\mathbf{3}}$ & $\mathbf{w}_{1}$ & $\mathbf{w}_{\mathbf{2}}$ & $\mathbf{w}_{\mathbf{3}}$ & $\mathbf{w}_{1}$ & $\mathbf{w}_{\mathbf{2}}$ & 0.0000 \\
0.1994 & 0.0000 & 0.8006 & 0.2188 & 0.0000 & 0.7812 & 0.2105 & 0.7895 \\
0.2261 & 0.1091 & 0.6648 & 0.2222 & 0.2304 & 0.5474 & 0.2095 & 0.1082 & 0.6823 \\
0.2381 & 0.2116 & 0.5503 & 0.2462 & 0.2745 & 0.4793 & 0.2737 & 0.1483 & 0.5780 \\
0.2639 & 0.2620 & 0.4741 & 0.2677 & 0.3050 & 0.4273 & 0.2865 & 0.2127 & 0.5008 \\
0.2620 & 0.2949 & 0.4431 & 0.2732 & 0.3241 & 0.4027 & 0.3241 & 0.2391 & 0.4368 \\
0.2750 & 0.3098 & 0.4152 & 0.2901 & 0.3338 & 0.3761 & 0.3319 & 0.2594 & 0.4087 \\
0.2844 & 0.3279 & 0.3876 & 0.8675 & 0.0000 & 0.1357 & 0.3403 & 0.2826 \\
0.3003 & 0.3456 & 0.3541 & 0.6259 & 0.2281 & 0.1460 & 0.3534 & 0.2881 & 0.3771 \\
0.3114 & 0.3666 & 0.3220 & 0.4576 & 0.3382 & 0.2043 & 0.3643 & 0.2962 & 0.3585 \\
0.7742 & 0.0840 & 0.1418 & 0.4033 & 0.3489 & 0.2478 & 0.3708 & 0.3035 \\
0.4397 & 0.4187 & 0.1416 & 0.3659 & 0.3768 & 0.2573 & 0.3792 & 0.3077 & 0.3258 \\
0.8955 & 0.0000 & 0.1045 & 0.3223 & 0.3642 & 0.3135 & 0.3845 & 0.3114 & 0.3130 \\
0.6001 & 0.3007 & 0.0992 & 0.4893 & 0.3256 & 0.1850 & 0.3906 & 0.3207 & 0.2887 \\
0.4962 & 0.3894 & 0.1144 & 0.4297 & 0.3721 & 0.1982 & 0.8865 & 0.0000 & 0.1135 \\
0.3499 & 0.4109 & 0.2391 & 0.2181 & 0.1802 & 0.6018 & 0.7855 & 0.0630 & 0.1515 \\
& & & 0.6966 & 0.1690 & 0.1344 & 0.6416 & 0.1888 & 0.1696 \\
& & & 0.7429 & 0.1089 & 0.1481 & 0.5425 & 0.2757 & 0.1818 \\
\hline
\end{tabular}

Titration uncertainty to a drop mass of butanol: $0.0052 \pm 0.0002 \mathrm{~g}$.

Titration uncertainty to a drop mass of water: $0.0151 \pm 0.00003 \mathrm{~g}$.

$\mathrm{u}(\mathrm{P})=0.1 \mathrm{kPa} ; \mathrm{u}(\mathrm{T})=0.1 \mathrm{~K} ; \mathrm{U}(\mathrm{w})=0.0008$ (aqueous phase) and $\mathrm{U}(\mathrm{w})=0.02$ (butanol phase)

Table 3: Binodal Curve data: Mass fraction (w) for Cloud Points of water (1) + m-2HEAB(2) + Butanol (3) Systems at $\mathrm{P}=101.2 \mathrm{kPa}$ and $293.15,313.15$ and $333.15 \mathrm{~K}$

\begin{tabular}{|c|c|c|c|c|c|c|c|c|}
\hline \multicolumn{3}{|c|}{ 293.15K } & \multicolumn{3}{|c|}{ 313.15K } & \multicolumn{3}{|c|}{ 333.15 } \\
\hline $\mathbf{w}_{1}$ & $\mathbf{w}_{2}$ & $\mathbf{w}_{3}$ & $\mathbf{w}_{1}$ & $\mathbf{w}_{2}$ & $\mathbf{w}_{3}$ & $\mathbf{w}_{1}$ & $\mathbf{w}_{2}$ & $\mathbf{w}_{3}$ \\
\hline 0.8809 & 0.0000 & 0.1191 & 0.2273 & 0.0000 & 0.7727 & 0.2533 & 0.0000 & 0.7467 \\
\hline 0.6012 & 0.1670 & 0.2318 & 0.2974 & 0.0491 & 0.6535 & 0.3645 & 0.0592 & 0.5763 \\
\hline 0.2158 & 0.0000 & 0.7842 & 0.3492 & 0.0757 & 0.5751 & 0.4338 & 0.0753 & 0.4909 \\
\hline 0.2677 & 0.0557 & 0.6767 & 0.4078 & 0.0949 & 0.4973 & 0.5009 & 0.0919 & 0.4071 \\
\hline 0.2287 & 0.1934 & 0.5780 & 0.4585 & 0.1075 & 0.4340 & 0.5370 & 0.0976 & 0.3654 \\
\hline 0.5475 & 0.1375 & 0.3150 & 0.4875 & 0.1139 & 0.3985 & 0.5695 & 0.1002 & 0.3303 \\
\hline 0.5352 & 0.1568 & 0.3080 & 0.5172 & 0.1176 & 0.3652 & 0.5976 & 0.1051 & 0.2973 \\
\hline 0.5685 & 0.1456 & 0.2860 & 0.5483 & 0.1236 & 0.3281 & 0.6192 & 0.1085 & 0.2723 \\
\hline 0.5956 & 0.1502 & 0.2542 & 0.5850 & 0.1256 & 0.2894 & 0.6395 & 0.1103 & 0.2502 \\
\hline 0.6090 & 0.1558 & 0.2352 & 0.6003 & 0.1329 & 0.2668 & 0.6523 & 0.1131 & 0.2346 \\
\hline 0.6005 & 0.1676 & 0.2319 & 0.6199 & 0.1355 & 0.2446 & 0.8150 & 0.0000 & 0.1850 \\
\hline 0.2729 & 0.0552 & 0.6719 & 0.6338 & 0.1367 & 0.2296 & 0.6641 & 0.1149 & 0.2211 \\
\hline 0.3647 & 0.0843 & 0.5510 & 0.6477 & 0.1383 & 0.2139 & 0.7538 & 0.0692 & 0.1770 \\
\hline 0.3879 & 0.0971 & 0.5149 & 0.6595 & 0.1397 & 0.2008 & & & \\
\hline 0.4255 & 0.1102 & 0.4643 & 0.6713 & 0.1405 & 0.1882 & & & \\
\hline 0.4643 & 0.1219 & 0.4138 & 0.6809 & 0.1413 & 0.1777 & & & \\
\hline 0.8330 & 0.0858 & 0.0812 & 0.9293 & 0.0000 & 0.0707 & & & \\
\hline 0.6632 & 0.1498 & 0.1869 & 0.8336 & 0.0857 & 0.0807 & & & \\
\hline 0.7814 & 0.1086 & 0.1100 & 0.7584 & 0.1326 & 0.1090 & & & \\
\hline 0.7602 & 0.1187 & 0.1211 & & & & & & \\
\hline 0.7443 & 0.1312 & 0.1245 & & & & & & \\
\hline
\end{tabular}

Titration uncertainty to a drop mass of butanol: $0.0052 \pm 0.0002 \mathrm{~g}$.

Titration uncertainty to a drop mass of water: $0.0151 \pm 0.00003 \mathrm{~g}$.

$\mathrm{u}(\mathrm{P})=0.1 \mathrm{kPa} ; \mathrm{u}(\mathrm{T})=0.1 \mathrm{~K} ; \mathrm{U}(\mathrm{w})=0.001$ (aqueous phase) and $\mathrm{U}(\mathrm{w})=0.003$ (butanol phase) 
Table 4: Binodal curve data: Mass fraction (w) of Cloud Points of water (1) + m-2HEAP (2) +Butanol (3) Systems at $\mathrm{P}=101.2 \mathrm{kPa}$ and $293.15,313.15$ and $333.15 \mathrm{~K}$

\begin{tabular}{|c|c|c|c|c|c|c|c|c|}
\hline \multicolumn{3}{|c|}{$293.15 K$} & \multicolumn{3}{|c|}{ 313.15K } & \multicolumn{3}{|c|}{333.15} \\
\hline $\mathbf{w}_{1}$ & $\mathbf{w}_{2}$ & $\mathbf{w}_{3}$ & $\mathbf{w}_{1}$ & $\mathbf{w}_{2}$ & $\mathbf{w}_{3}$ & $\mathbf{w}_{1}$ & $\mathbf{w}_{2}$ & $\mathbf{w}_{3}$ \\
\hline 0.2300 & 0.0000 & 0.7700 & 0.2896 & 0.0000 & 0.7104 & 0.2666 & 0.0000 & 0.7334 \\
\hline 0.3064 & 0.0359 & 0.6576 & 0.5247 & 0.0639 & 0.4114 & 0.3893 & 0.0408 & 0.5699 \\
\hline 0.3999 & 0.0530 & 0.5472 & 0.6077 & 0.0706 & 0.3217 & 0.4926 & 0.0550 & 0.4523 \\
\hline 0.4668 & 0.0664 & 0.4668 & 0.6646 & 0.0753 & 0.2601 & 0.5753 & 0.0623 & 0.3624 \\
\hline 0.5657 & 0.0752 & 0.3591 & 0.6997 & 0.0757 & 0.2246 & 0.6199 & 0.0710 & 0.3091 \\
\hline 0.6369 & 0.0810 & 0.2821 & 0.7357 & 0.0763 & 0.1880 & 0.6677 & 0.0686 & 0.2636 \\
\hline 0.6710 & 0.0821 & 0.2469 & 0.7570 & 0.0762 & 0.1668 & 0.8456 & 0.0000 & 0.1544 \\
\hline 0.6921 & 0.0829 & 0.2250 & 0.7731 & 0.0757 & 0.1512 & 0.7594 & 0.0604 & 0.1802 \\
\hline 0.9214 & 0.0000 & 0.0786 & 0.7898 & 0.0745 & 0.1357 & 0.7990 & 0.0330 & 0.1680 \\
\hline 0.8308 & 0.0439 & 0.1252 & 0.8943 & 0.0000 & 0.1057 & 0.7323 & 0.0667 & 0.2010 \\
\hline 0.6153 & 0.0877 & 0.2970 & 0.8942 & 0.0000 & 0.1058 & & & \\
\hline 0.8148 & 0.0530 & 0.1322 & 0.8800 & 0.0275 & 0.0924 & & & \\
\hline 0.7970 & 0.0643 & 0.1387 & 0.4261 & 0.0468 & 0.5271 & & & \\
\hline 0.7225 & 0.0804 & 0.1971 & 0.3423 & 0.0284 & 0.6292 & & & \\
\hline 0.8664 & 0.0207 & 0.1129 & 0.8561 & 0.0523 & 0.0916 & & & \\
\hline
\end{tabular}

Titration uncertainty to a drop mass of butanol: $0.0052 \pm 0.0002 \mathrm{~g}$.

Titration uncertainty to a drop mass of water: $0.0151 \pm 0.00003 \mathrm{~g}$.

$\mathrm{u}(\mathrm{P})=0.1 \mathrm{kPa} ; \mathrm{u}(\mathrm{T})=0.1 \mathrm{~K} ; \mathrm{U}(\mathrm{w})=0.001$ (aqueous phase) and $\mathrm{U}(\mathrm{w})=0.003$ (butanol phase)

Table 5: Binodal curve data: Mass fraction (w) of Cloud Points of water (1) + m-2HEAPr (2) +Pentanol (3) Systems at $\mathrm{P}=101.2 \mathrm{kPa}$ and $293.15,313.15$ and $333.15 \mathrm{~K}$

\begin{tabular}{|c|c|c|c|c|c|c|c|c|}
\hline \multicolumn{3}{|c|}{$293.15 K$} & \multicolumn{3}{|c|}{ 313.15K } & \multicolumn{3}{|c|}{333.15} \\
\hline$w_{1}$ & $\mathbf{w}_{2}$ & $\mathbf{w}_{3}$ & $w_{1}$ & $\mathrm{w}_{2}$ & $\mathbf{w}_{3}$ & $w_{1}$ & $\mathbf{w}_{2}$ & $\mathbf{w}_{3}$ \\
\hline 0.1254 & 0.0000 & 0.8746 & 0.1086 & 0.0000 & 0.8914 & 0.1476 & 0.0000 & 0.8524 \\
\hline 0.1688 & 0.1034 & 0.7278 & 0.1526 & 0.0908 & 0.7566 & 0.1781 & 0.1051 & 0.7168 \\
\hline 0.1849 & 0.1434 & 0.6717 & 0.2010 & 0.1402 & 0.6587 & 0.2163 & 0.1644 & 0.6194 \\
\hline 0.2120 & 0.1724 & 0.6156 & 0.2384 & 0.1787 & 0.5829 & 0.2736 & 0.1979 & 0.5285 \\
\hline 0.2308 & 0.2097 & 0.5595 & 0.2624 & 0.2255 & 0.5121 & 0.3098 & 0.2406 & 0.4496 \\
\hline 0.2667 & 0.2392 & 0.4941 & 0.2805 & 0.2457 & 0.4738 & 0.3331 & 0.2509 & 0.4160 \\
\hline 0.2813 & 0.2578 & 0.4610 & 0.2911 & 0.2574 & 0.4515 & 0.3484 & 0.2693 & 0.3822 \\
\hline 0.2855 & 0.2762 & 0.4383 & 0.3118 & 0.2793 & 0.4089 & 0.3653 & 0.2807 & 0.3540 \\
\hline 0.2994 & 0.2846 & 0.4160 & 0.3238 & 0.2922 & 0.3840 & 0.3808 & 0.2886 & 0.3306 \\
\hline 0.3044 & 0.2968 & 0.3988 & 0.3421 & 0.3067 & 0.3512 & 0.3840 & 0.3100 & 0.3061 \\
\hline 0.3127 & 0.3067 & 0.3807 & 0.3523 & 0.3177 & 0.3300 & 0.3966 & 0.3079 & 0.2955 \\
\hline 0.3202 & 0.3185 & 0.3614 & 0.9638 & 0.0097 & 0.0264 & 0.4019 & 0.3250 & 0.2732 \\
\hline 0.3342 & 0.3258 & 0.3400 & 0.8214 & 0.1408 & 0.0378 & 0.4141 & 0.3494 & 0.2366 \\
\hline 0.3408 & 0.3338 & 0.3254 & 0.7460 & 0.2222 & 0.0319 & 0.4288 & 0.3721 & 0.1991 \\
\hline 0.9061 & 0.0692 & 0.0248 & 0.7253 & 0.2421 & 0.0326 & 0.9599 & 0.0185 & 0.0216 \\
\hline 0.8583 & 0.1182 & 0.0235 & 0.7203 & 0.2446 & 0.0352 & 0.9017 & 0.0703 & 0.0280 \\
\hline 0.4198 & 0.5728 & 0.0074 & 0.9516 & 0.0196 & 0.0288 & 0.7726 & 0.1957 & 0.0317 \\
\hline 0.7822 & 0.2008 & 0.0170 & 0.6880 & 0.2807 & 0.0312 & 0.6658 & 0.2978 & 0.0364 \\
\hline 0.9568 & 0.0207 & 0.0226 & 0.6005 & 0.3649 & 0.0346 & 0.5986 & 0.3562 & 0.0452 \\
\hline 0.9371 & 0.0408 & 0.0221 & 0.5284 & 0.4169 & 0.0547 & 0.5496 & 0.3871 & 0.0633 \\
\hline 0.8982 & 0.0777 & 0.0242 & 0.4820 & 0.4232 & 0.0949 & 0.5214 & 0.3912 & 0.0874 \\
\hline 0.6391 & 0.3335 & 0.0274 & 0.4267 & 0.4090 & 0.1642 & 0.5013 & 0.3908 & 0.1078 \\
\hline 0.5170 & 0.4378 & 0.0451 & 0.3846 & 0.3825 & 0.2329 & 0.4880 & 0.3895 & 0.1225 \\
\hline 0.4289 & 0.4618 & 0.1094 & & & & 0.4831 & 0.3956 & 0.1213 \\
\hline 0.4134 & 0.4528 & 0.1339 & & & & & & \\
\hline 0.4021 & 0.4447 & 0.1532 & & & & & & \\
\hline 0.3660 & 0.3914 & 0.2426 & & & & & & \\
\hline
\end{tabular}

Titration uncertainty to a drop mass of pentanol: $0.0063 \pm 0.00004 \mathrm{~g}$. Titration uncertainty to a drop mass of water: $0.0151 \pm 0.00003 \mathrm{~g} . \mathrm{u}(\mathrm{P})=0.1 \mathrm{kPa}$; $\mathrm{u}(\mathrm{T})=0.1 \mathrm{~K} ; \mathrm{U}(\mathrm{w})=0.004$ (aqueous phase) and $\mathrm{U}(\mathrm{w})=0.001$ (pentanol phase) 
Table 6: Binodal curve data: Mass fraction (w) of Cloud Points of water (1) + m- 2HEAB (2) +Pentanol (3) Systems at $\mathrm{P}=101.2 \mathrm{kPa}$ and $293.15,313.15$ and $333.15 \mathrm{~K}$

\begin{tabular}{|c|c|c|c|c|c|c|c|c|}
\hline \multicolumn{3}{|c|}{$293.15 K$} & \multicolumn{3}{|c|}{$313.15 K$} & \multicolumn{3}{|c|}{333.15} \\
\hline$w_{1}$ & $\mathbf{w}_{2}$ & $\mathbf{w}_{3}$ & $w_{1}$ & $\mathbf{w}_{2}$ & $\mathbf{w}_{3}$ & $w_{1}$ & $\mathbf{w}_{2}$ & $\mathbf{w}_{3}$ \\
\hline 0.0665 & 0.0000 & 0.9335 & 0.0603 & 0.0000 & 0.9397 & 0.2077 & 0.0000 & 0.7923 \\
\hline 0.0989 & 0.0361 & 0.8650 & 0.1687 & 0.0863 & 0.7451 & 0.3015 & 0.0441 & 0.6544 \\
\hline 0.1241 & 0.0627 & 0.8132 & 0.3051 & 0.1296 & 0.5653 & 0.3241 & 0.0645 & 0.6114 \\
\hline 0.1746 & 0.1060 & 0.7193 & 0.3493 & 0.1587 & 0.4920 & 0.3644 & 0.1155 & 0.5201 \\
\hline 0.2082 & 0.1302 & 0.6616 & 0.3964 & 0.1755 & 0.4281 & 0.3921 & 0.1344 & 0.4735 \\
\hline 0.2240 & 0.1487 & 0.6273 & 0.4065 & 0.1926 & 0.4009 & 0.4188 & 0.1457 & 0.4356 \\
\hline 0.2862 & 0.1867 & 0.5272 & 0.4415 & 0.2047 & 0.3538 & 0.4294 & 0.1577 & 0.4129 \\
\hline 0.2842 & 0.2033 & 0.5126 & 0.4630 & 0.2111 & 0.3259 & 0.4483 & 0.1673 & 0.3844 \\
\hline 0.3105 & 0.2001 & 0.4895 & 0.4773 & 0.2193 & 0.3035 & 0.4892 & 0.1846 & 0.3262 \\
\hline 0.3281 & 0.2075 & 0.4644 & 0.4989 & 0.2231 & 0.2779 & 0.5161 & 0.1996 & 0.2843 \\
\hline 0.3426 & 0.2161 & 0.4413 & 0.5132 & 0.2268 & 0.2600 & 0.5216 & 0.2014 & 0.2771 \\
\hline 0.3573 & 0.2264 & 0.4163 & 0.5209 & 0.2352 & 0.2439 & 0.5263 & 0.2045 & 0.2692 \\
\hline 0.3705 & 0.2317 & 0.3978 & 0.5357 & 0.2372 & 0.2271 & 0.5343 & 0.2085 & 0.2572 \\
\hline 0.3835 & 0.2399 & 0.3767 & 0.5466 & 0.2410 & 0.2124 & 0.5522 & 0.2125 & 0.2353 \\
\hline 0.3964 & 0.2451 & 0.3585 & 0.5567 & 0.2420 & 0.2013 & 0.5552 & 0.2157 & 0.2291 \\
\hline 0.4070 & 0.2481 & 0.3450 & 0.5641 & 0.2468 & 0.1891 & 0.5674 & 0.2160 & 0.2165 \\
\hline 0.4175 & 0.2527 & 0.3298 & 0.5708 & 0.2480 & 0.1812 & 0.5759 & 0.2195 & 0.2046 \\
\hline 0.4322 & 0.2568 & 0.3110 & 0.9646 & 0.0000 & 0.0354 & 0.5832 & 0.2218 & 0.1950 \\
\hline 0.4438 & 0.2614 & 0.2947 & 0.9550 & 0.0230 & 0.0220 & 0.9623 & 0.0131 & 0.0245 \\
\hline 0.4523 & 0.2656 & 0.2820 & 0.8251 & 0.1652 & 0.0097 & 0.9355 & 0.0334 & 0.0311 \\
\hline 0.4655 & 0.2696 & 0.2648 & 0.8040 & 0.1748 & 0.0212 & 0.7675 & 0.1977 & 0.0348 \\
\hline 0.4714 & 0.2732 & 0.2554 & 0.7228 & 0.2516 & 0.0256 & 0.7362 & 0.2147 & 0.0491 \\
\hline 0.4835 & 0.2771 & 0.2395 & 0.7114 & 0.2603 & 0.0282 & 0.6477 & 0.2280 & 0.1243 \\
\hline 0.4938 & 0.2802 & 0.2261 & 0.6391 & 0.2848 & 0.0761 & 0.8117 & 0.1589 & 0.0294 \\
\hline 0.5021 & 0.2835 & 0.2143 & 0.6774 & 0.2744 & 0.0482 & 0.8786 & 0.0886 & 0.0328 \\
\hline 0.5157 & 0.2869 & 0.1975 & 0.8871 & 0.1061 & 0.0068 & & & \\
\hline 0.5185 & 0.2886 & 0.1929 & 0.6149 & 0.2877 & 0.0974 & & & \\
\hline 0.5340 & 0.2931 & 0.1729 & 0.5860 & 0.2674 & 0.1466 & & & \\
\hline 0.6734 & 0.2472 & 0.0794 & & & & & & \\
\hline 0.6083 & 0.2876 & 0.1040 & & & & & & \\
\hline 0.9596 & 0.0000 & 0.0404 & & & & & & \\
\hline 0.8311 & 0.1083 & 0.0606 & & & & & & \\
\hline 0.7234 & 0.2127 & 0.0639 & & & & & & \\
\hline 0.5126 & 0.2852 & 0.2022 & & & & & & \\
\hline 0.5770 & 0.3008 & 0.1222 & & & & & & \\
\hline 0.5578 & 0.2938 & 0.1485 & & & & & & \\
\hline 0.7697 & 0.1635 & 0.0668 & & & & & & \\
\hline
\end{tabular}

Titration uncertainty to a drop mass of pentanol: $0.0063 \pm 0.00004 \mathrm{~g}$.

Titration uncertainty to a drop mass of water: $0.0151 \pm 0.00003 \mathrm{~g}$.

$\mathrm{u}(\mathrm{P})=0.1 \mathrm{kPa} ; \mathrm{u}(\mathrm{T})=0.1 \mathrm{~K} ; \mathrm{U}(\mathrm{w})=0.004$ (aqueous phase) and $\mathrm{U}(\mathrm{w})=0.01$ (pentanol phase) 
Table 7: Binodal curve data: Mass fraction (w) of Cloud Points of water (1) + m-2HEAP (2) + Pentanol (3) Systems at $\mathrm{P}=101.2 \mathrm{kPa}$ and $293.15,313.15$ and $333.15 \mathrm{~K}$.

\begin{tabular}{|c|c|c|c|c|c|c|c|c|}
\hline \multicolumn{3}{|c|}{$293.15 K$} & \multicolumn{3}{|c|}{ 313.15K } & \multicolumn{3}{|c|}{333.15} \\
\hline$w_{1}$ & $w_{2}$ & $w_{3}$ & $w_{1}$ & $w_{2}$ & $\mathbf{w}_{3}$ & $w_{1}$ & $w_{2}$ & $w_{3}$ \\
\hline 0.1187 & 0.0000 & 0.8813 & 0.0834 & 0.0000 & 0.9166 & 0.1763 & 0.0000 & 0.8237 \\
\hline 0.2548 & 0.0694 & 0.6758 & 0.1574 & 0.0512 & 0.7914 & 0.2537 & 0.1047 & 0.6417 \\
\hline 0.3208 & 0.0970 & 0.5821 & 0.2696 & 0.0787 & 0.6517 & 0.3232 & 0.1291 & 0.5476 \\
\hline 0.3650 & 0.1140 & 0.5210 & 0.3019 & 0.0925 & 0.6056 & 0.3660 & 0.1424 & 0.4916 \\
\hline 0.4168 & 0.1343 & 0.4489 & 0.3548 & 0.1110 & 0.5343 & 0.4105 & 0.1498 & 0.4397 \\
\hline 0.4606 & 0.1456 & 0.3938 & 0.3948 & 0.1185 & 0.4867 & 0.4544 & 0.1571 & 0.3885 \\
\hline 0.4897 & 0.1524 & 0.3579 & 0.4208 & 0.1257 & 0.4535 & 0.4892 & 0.1612 & 0.3496 \\
\hline 0.5174 & 0.1555 & 0.3271 & 0.4430 & 0.1299 & 0.4271 & 0.5211 & 0.1621 & 0.3168 \\
\hline 0.9114 & 0.0690 & 0.0197 & 0.4908 & 0.1387 & 0.3705 & 0.5698 & 0.1622 & 0.2680 \\
\hline 0.9787 & 0.0062 & 0.0150 & 0.5144 & 0.1413 & 0.3443 & 0.5991 & 0.1583 & 0.2426 \\
\hline 0.8175 & 0.1614 & 0.0211 & 0.5581 & 0.1458 & 0.2961 & 0.6733 & 0.1643 & 0.1625 \\
\hline 0.7959 & 0.1750 & 0.0290 & 0.5775 & 0.1467 & 0.2758 & 0.7066 & 0.1581 & 0.1353 \\
\hline 0.7545 & 0.1828 & 0.0627 & 0.5872 & 0.1498 & 0.2630 & 0.7108 & 0.1588 & 0.1304 \\
\hline 0.7325 & 0.1862 & 0.0813 & 0.6024 & 0.1512 & 0.2464 & 0.9620 & 0.0119 & 0.0261 \\
\hline 0.7026 & 0.1878 & 0.1096 & 0.6140 & 0.1525 & 0.2335 & 0.8508 & 0.1151 & 0.0341 \\
\hline 0.6646 & 0.1881 & 0.1473 & 0.6287 & 0.1536 & 0.2177 & 0.8158 & 0.1428 & 0.0414 \\
\hline 0.6443 & 0.1875 & 0.1682 & 0.6401 & 0.1547 & 0.2052 & 0.7778 & 0.1534 & 0.0688 \\
\hline 0.6095 & 0.1868 & 0.2036 & 0.6470 & 0.1562 & 0.1969 & 0.6503 & 0.1600 & 0.1897 \\
\hline 0.5748 & 0.1850 & 0.2402 & 0.6569 & 0.1565 & 0.1866 & 0.6058 & 0.1585 & 0.2358 \\
\hline \multirow[t]{6}{*}{0.3526} & 0.1201 & 0.5273 & 0.6695 & 0.1566 & 0.1739 & 0.5725 & 0.1565 & 0.2710 \\
\hline & & & 0.8066 & 0.1603 & 0.0331 & & & \\
\hline & & & 0.7884 & 0.1566 & 0.0550 & & & \\
\hline & & & 0.7791 & 0.1577 & 0.0632 & & & \\
\hline & & & 0.8234 & 0.1481 & 0.0285 & & & \\
\hline & & & 0.7462 & 0.1643 & 0.0894 & & & \\
\hline
\end{tabular}

Titration uncertainty to a drop mass of pentanol: $0.0063 \pm 0.00004 \mathrm{~g}$.

Titration uncertainty to a drop mass of water: $0.0151 \pm 0.00003 \mathrm{~g}$.

$\mathrm{u}(\mathrm{P})=0.1 \mathrm{kPa} ; \mathrm{u}(\mathrm{T})=0.1 \mathrm{~K} ; \mathrm{U}(\mathrm{w})=0.004$ (aqueous phase) and $\mathrm{U}(\mathrm{w})=0.01$ (pentanol phase)

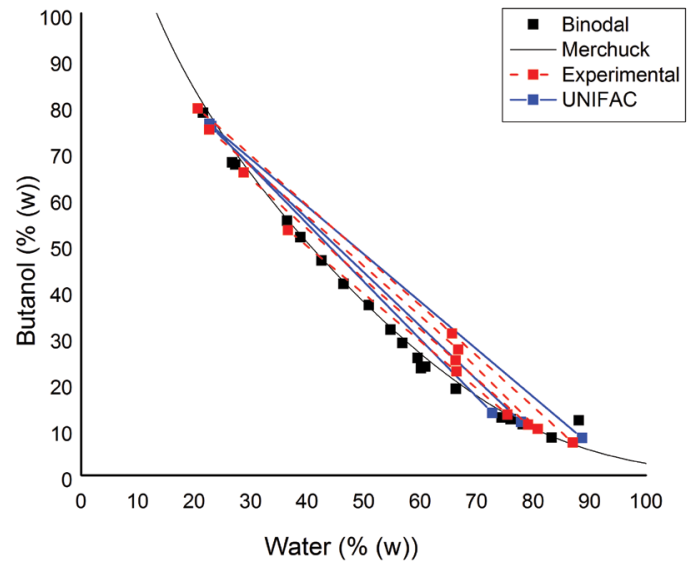

Figure 7: Tie-line data for water $+\mathrm{m}-2 \mathrm{HEAB}+$ butanol at $293.15 \mathrm{~K}$ UNIFAC model (continuous lines).

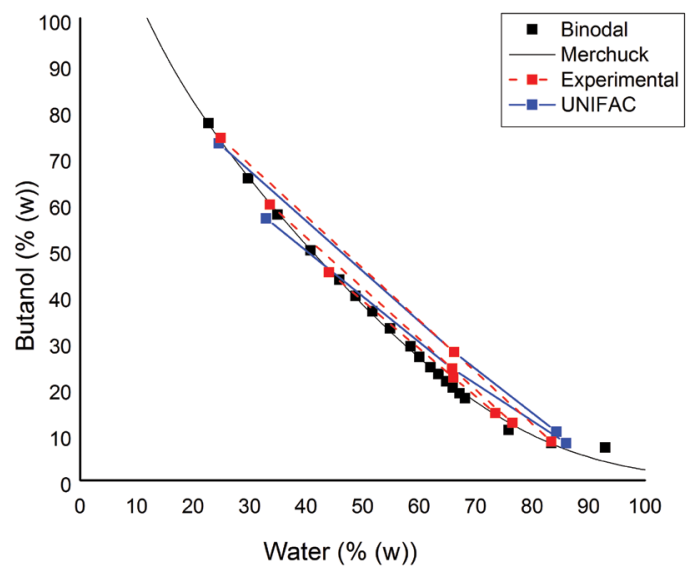

Figure 8: Tie-line data for water + m-2HEAB + butanol at $313.15 \mathrm{~K}$ UNIFAC model (continuous lines). 


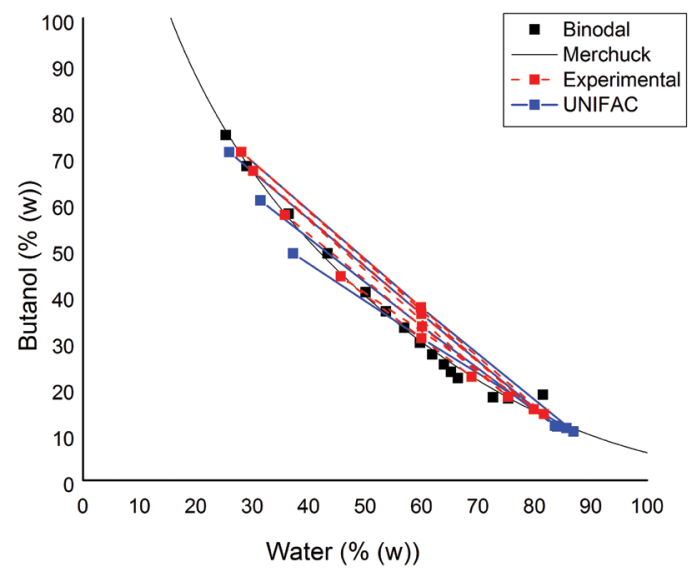

Figure 9: Tie-line datas to water $+\mathrm{m}-2 \mathrm{HEAB}+$ butanol at $333.15 \mathrm{~K}$, UNIFAC model (continuous lines).

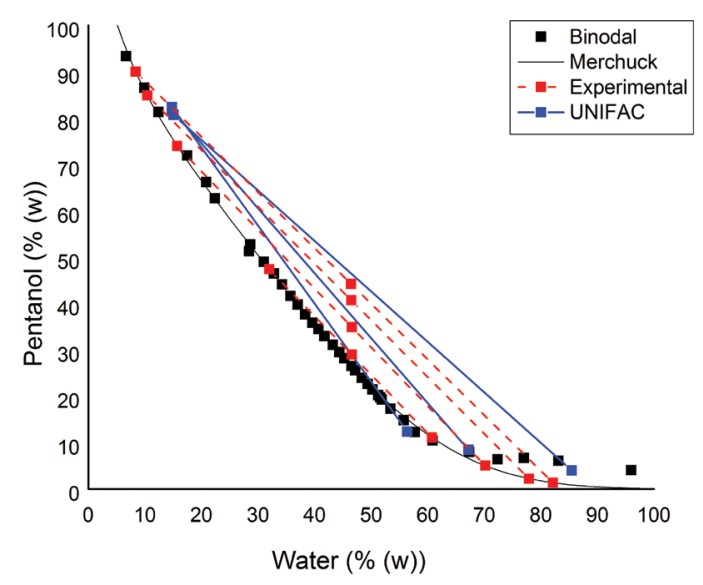

Figure 10: Tie-line data for water $+\mathrm{m}-2 \mathrm{HEAB}+$ pentanol at $293.15 \mathrm{~K}$, UNIFAC model (continuous lines).

The binodal curves were fitted according to Merchuk et al. (1998). After the identification of miscible and immiscible regions, it is possible to determine the LLE tie- lines with the measured weight of each phase in equilibrium Tables 8-9 show the mass composition of each studied tie-line. Partition coefficients of the ionic liquid in alcohol-water systems are gathered in Table 10.

The consistency of the measured tie-lines was checked using Hand (1930) and Othmer and Tobias (1942) correlations:

$$
\log \frac{\left(x_{2}^{\text {water }}\right)}{\left(x_{1}^{\text {water }}\right)}=k_{H} \log \frac{\left(x_{2}^{\text {alcohol }}\right)}{\left(x_{3}^{\text {alcohol }}\right)}+\operatorname{const}_{H}
$$

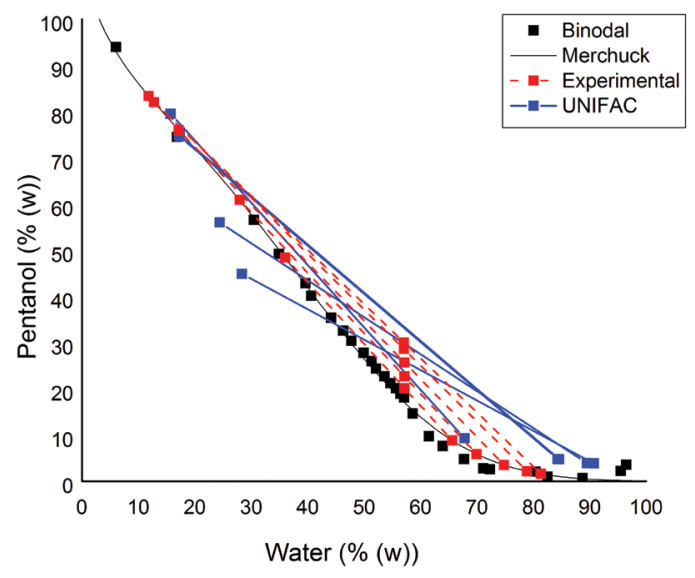

Figure 11: Tie-line data for water $+\mathrm{m}-2 \mathrm{HEAB}+$ pentanol at $313.15 \mathrm{~K}$, UNIFAC model (continuous lines).

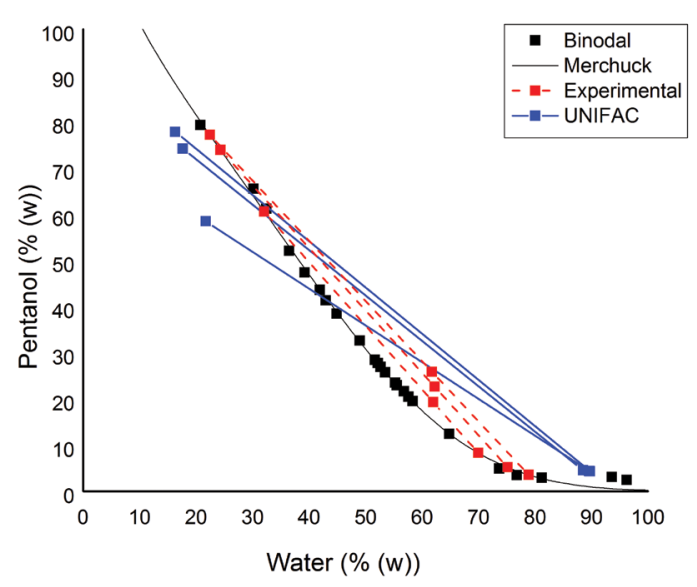

Figure 12. Tie-line data for water $+\mathrm{m}-2 \mathrm{HEAB}+$ pentanol at $333.15 \mathrm{~K}$, UNIFAC model (continuous lines).

$\log \frac{\left(1-x_{1}^{\text {water }}\right)}{\left(x_{1}^{\text {water }}\right)}=k_{O T} \log \frac{\left(1-\chi_{3}^{\text {alcohol }}\right)}{\left(x_{3}^{\text {alcohol })}\right)}+$ const $_{O T}$

where $x_{2}^{\text {water }}, x_{1}^{\text {water }}, x_{2}^{\text {alcohol }}, x_{3}^{\text {alcohol }}$, are the mole fraction of the IL in the water-rich phase, water in the water-rich phase, IL in the alcohol-rich phase and alcohol in the alcohol-rich phase, respectively, and $k_{\mathrm{H}}$, const $_{\mathrm{H}}, k_{\mathrm{OT}}$, and const ${ }_{\mathrm{OH}}$ are the constants obtained by linear data fit. It is worth mentioning that water was considered as solvent, IL as solute and alcohol as diluent. The results are shown in Tables 10 and 11. Data were well correlated as $\mathrm{R}^{2}$ for the majority of the data was above 0.8 . However, some systems such as water + m-2HEAPr +1 -butanol at $313 \mathrm{~K}$ failed the Hand test but passed the Othmer-Tobias test, water $+\mathrm{m}-2 \mathrm{HEAB}$ 
+ 1-pentanol at 333.15 passed the Hand test but failed the Othmer-Tobias test and water $+\mathrm{m}-2 \mathrm{HEAP}+1$-butanol, at $333.15 \mathrm{~K}$ failed both tests.

Table 8: Tie-Lines of water (1) + IL (2) + butanol (3), weight fractions in the Top (T) and Bottom (B) Phases and at the Initial Biphasic Composition of the Mixture (M) and $101.2 \mathrm{kPa}$

\begin{tabular}{|c|c|c|c|c|c|c|c|c|c|}
\hline \multicolumn{10}{|c|}{ 293.15K } \\
\hline $\mathrm{IL}$ & $w_{1}^{T}$ & $w_{2}{ }^{T}$ & $w_{3}^{T}$ & $w_{1}^{M}$ & $w_{2}^{M}$ & $w_{3}{ }^{M}$ & $w_{1}{ }^{B}$ & $w_{2}^{B}$ & $w_{3}^{B}$ \\
\hline \multirow{3}{*}{$\mathrm{m}$-2HEAPr } & 0.1713 & 0.1549 & 0.6738 & 0.4440 & 0.1942 & 0.3618 & 0.6721 & 0.2270 & 0.1008 \\
\hline & 0.1479 & 0.0456 & 0.8065 & 0.4420 & 0.1396 & 0.4183 & 0.6830 & 0.2170 & 0.0999 \\
\hline & 0.1344 & 0.0640 & 0.8016 & 0.4401 & 0.1133 & 0.4465 & 0.6724 & 0.2268 & 0.1008 \\
\hline \multirow{4}{*}{ m-2HEAB } & 0.3662 & 0.1035 & 0.5303 & 0.6643 & 0.1114 & 0.2244 & 0.7548 & 0.1138 & 0.1314 \\
\hline & 0.2875 & 0.0576 & 0.6549 & 0.6629 & 0.0887 & 0.2484 & 0.7912 & 0.0993 & 0.1095 \\
\hline & 0.2267 & 0.0255 & 0.7478 & 0.6624 & 0.0554 & 0.2822 & 0.8086 & 0.0915 & 0.0999 \\
\hline & 0.2063 & 0.0003 & 0.7935 & 0.6566 & 0.0367 & 0.3068 & 0.8702 & 0.0591 & 0.0707 \\
\hline \multirow{4}{*}{ m-2HEAP } & 0.4633 & 0.0713 & 0.4653 & 0.6674 & 0.0667 & 0.2659 & 0.7693 & 0.0644 & 0.1663 \\
\hline & 0.3457 & 0.0451 & 0.6093 & 0.6673 & 0.0456 & 0.2871 & 0.8280 & 0.0459 & 0.1262 \\
\hline & 0.2251 & 0.0438 & 0.7311 & 0.6509 & 0.0218 & 0.3273 & 0.8547 & 0.0352 & 0.1101 \\
\hline & 0.2410 & 0.0030 & 0.7560 & 0.6664 & 0.0115 & 0.3221 & 0.8957 & 0.0161 & 0.0882 \\
\hline \multicolumn{10}{|c|}{$313.15 \mathrm{~K}$} \\
\hline IL & $w_{1}^{T}$ & $w_{2}{ }^{T}$ & $w_{3}{ }^{T}$ & $w_{1}^{M}$ & $w_{2}^{M}$ & $w_{3}{ }^{M}$ & $w_{1}^{B}$ & $w_{2}^{B}$ & $w_{3}{ }^{B}$ \\
\hline \multirow{4}{*}{ m-2HEAPr } & 0.1618 & 0.0763 & 0.9151 & 0.4435 & 0.0834 & 0.4731 & 0.6632 & 0.2076 & 0.1292 \\
\hline & 0.5072 & 0.0038 & 0.4889 & 0.5042 & 0.0955 & 0.4003 & 0.4889 & 0.0248 & 0.4863 \\
\hline & 0.4856 & 0.0254 & 0.4889 & 0.5067 & 0.1429 & 0.3505 & 0.4889 & 0.0927 & 0.4184 \\
\hline & 0.4715 & 0.0395 & 0.4890 & 0.5087 & 0.1914 & 0.2999 & 0.4890 & 0.1681 & 0.3429 \\
\hline \multirow{3}{*}{$\mathrm{m}-2 \mathrm{HEAB}$} & 0.4404 & 0.1097 & 0.4500 & 0.6605 & 0.1173 & 0.2222 & 0.7351 & 0.1198 & 0.1451 \\
\hline & 0.3359 & 0.0674 & 0.5967 & 0.6584 & 0.1000 & 0.2416 & 0.7649 & 0.1108 & 0.1243 \\
\hline & 0.2487 & 0.0106 & 0.7408 & 0.6617 & 0.0611 & 0.2772 & 0.8339 & 0.0822 & 0.0839 \\
\hline \multirow{4}{*}{ m-2HEAP } & 0.5167 & 0.0719 & 0.4113 & 0.6981 & 0.0694 & 0.2325 & 0.7603 & 0.0685 & 0.1712 \\
\hline & 0.391 & 0.0405 & 0.5685 & 0.7186 & 0.0473 & 0.2341 & 0.8247 & 0.0494 & 0.1259 \\
\hline & 0.3385 & 0.0208 & 0.6407 & 0.7024 & 0.0364 & 0.2612 & 0.843 & 0.0425 & 0.1145 \\
\hline & 0.2714 & 0.0169 & 0.7117 & 0.7061 & 0.0242 & 0.2697 & 0.856 & 0.0371 & 0.1069 \\
\hline \multicolumn{10}{|c|}{ 333.15K } \\
\hline IL & $w_{1}^{T}$ & $w_{2}^{T}$ & $w_{3}{ }^{T}$ & $w_{1}^{M}$ & $w_{2}{ }^{M}$ & $w_{3}{ }^{M}$ & $w_{1}^{B}$ & $w_{2}^{B}$ & $w_{3}^{B}$ \\
\hline \multirow{4}{*}{ m-2HEAPr } & 0.1918 & 0.1036 & 0.7045 & 0.4995 & 0.1264 & 0.3741 & 0.7126 & 0.1422 & 0.1451 \\
\hline & 0.1770 & 0.0629 & 0.7601 & 0.4995 & 0.0630 & 0.4376 & 0.8108 & 0.0630 & 0.1262 \\
\hline & 0.3790 & 0.1163 & 0.5047 & 0.4407 & 0.1174 & 0.4419 & 0.5023 & 0.1186 & 0.3790 \\
\hline & 0.3576 & 0.1022 & 0.5402 & 0.4541 & 0.0970 & 0.4489 & 0.5505 & 0.0919 & 0.3576 \\
\hline \multirow{4}{*}{ m-2HEAB } & 0.3580 & 0.0677 & 0.5743 & 0.6008 & 0.0669 & 0.3324 & 0.7531 & 0.0664 & 0.1805 \\
\hline & 0.3019 & 0.0291 & 0.6690 & 0.5996 & 0.0404 & 0.3600 & 0.7986 & 0.0479 & 0.1534 \\
\hline & 0.2804 & 0.0093 & 0.7103 & 0.5987 & 0.0273 & 0.3740 & 0.8171 & 0.0396 & 0.1433 \\
\hline & 0.4566 & 0.1025 & 0.4409 & 0.5996 & 0.0931 & 0.3073 & 0.6888 & 0.0873 & 0.2239 \\
\hline \multirow{3}{*}{ 2HEAP } & 0.7029 & 0.0220 & 0.2751 & 0.7000 & 0.0101 & 0.2899 & 0.2751 & 0.4394 & 0.2856 \\
\hline & 0.3570 & 0.0090 & 0.6340 & 0.6515 & 0.0667 & 0.2817 & 0.8426 & 0.0357 & 0.1217 \\
\hline & 0.3335 & 0.0013 & 0.6653 & 0.6617 & 0.0237 & 0.3146 & 0.9045 & 0.005 & 0.0905 \\
\hline
\end{tabular}


Table 9: Weight Fraction Compositions for TLs of water (1) + IL (2) + pentanol (3), in the Top (T) and Bottom (B) Phases and at the Initial Biphasic Composition of the Mixture (M) and $101.2 \mathrm{kPa}$

\begin{tabular}{|c|c|c|c|c|c|c|c|c|c|}
\hline \multicolumn{10}{|c|}{ 293.15K } \\
\hline IL & $w_{1}^{T}$ & $w_{2}^{T}$ & $w_{3}{ }^{T}$ & $w_{1}^{M}$ & $w_{2}^{M}$ & $w_{3}^{M}$ & $w_{1}^{B}$ & $w_{2}^{B}$ & $w_{3}^{B}$ \\
\hline \multirow{4}{*}{ m-2HEAPr } & 0.5532 & 0.1979 & 0.2489 & 0.6982 & 0.0277 & 0.2740 & 0.2489 & 0.3087 & 0.4424 \\
\hline & 0.5529 & 0.1963 & 0.2507 & 0.6995 & 0.0506 & 0.2499 & 0.2507 & 0.3590 & 0.3903 \\
\hline & 0.1816 & 0.0288 & 0.7896 & 0.5915 & 0.0415 & 0.3670 & 0.8379 & 0.0967 & 0.0653 \\
\hline & 0.1683 & 0.0284 & 0.8033 & 0.5978 & 0.0062 & 0.3960 & 0.8889 & 0.0592 & 0.0518 \\
\hline \multirow{4}{*}{ m-2HEAB } & 0.3199 & 0.2063 & 0.4737 & 0.4657 & 0.2446 & 0.2897 & 0.6075 & 0.2818 & 0.1107 \\
\hline & 0.1572 & 0.1032 & 0.7395 & 0.4658 & 0.1854 & 0.3488 & 0.7017 & 0.2482 & 0.0501 \\
\hline & 0.1043 & 0.0472 & 0.8485 & 0.4650 & 0.1282 & 0.4068 & 0.7790 & 0.1986 & 0.0224 \\
\hline & 0.0837 & 0.0164 & 0.8999 & 0.4645 & 0.0933 & 0.4422 & 0.8212 & 0.1653 & 0.0135 \\
\hline \multirow{3}{*}{ m-2HEAP } & 0.1443 & 0.0472 & 0.8085 & 0.4650 & 0.1282 & 0.4068 & 0.7990 & 0.1386 & 0.0624 \\
\hline & 0.0707 & 0.0094 & 0.9199 & 0.4645 & 0.0933 & 0.4422 & 0.8212 & 0.1253 & 0.0535 \\
\hline & 0.0359 & 0.0085 & 0.9557 & 0.4641 & 0.0601 & 0.4758 & 0.8501 & 0.1124 & 0.0376 \\
\hline \multicolumn{10}{|c|}{$313.15 K$} \\
\hline IL & $w_{1}^{T}$ & $w_{2}{ }^{T}$ & $w_{3}^{T}$ & $w_{1}^{M}$ & $w_{2}^{M}$ & $w_{3}{ }^{M}$ & $w_{1}^{B}$ & $w_{2}^{B}$ & $w_{3}{ }^{B}$ \\
\hline \multirow{4}{*}{ m-2HEAPr } & 0.5725 & 0.0162 & 0.4113 & 0.3995 & 0.2007 & 0.3998 & 0.4113 & 0.2018 & 0.3869 \\
\hline & 0.5612 & 0.0288 & 0.4100 & 0.3999 & 0.1501 & 0.4500 & 0.4100 & 0.3250 & 0.2650 \\
\hline & 0.0816 & 0.0188 & 0.8996 & 0.5815 & 0.0515 & 0.3670 & 0.8479 & 0.0857 & 0.0663 \\
\hline & 0.0883 & 0.0084 & 0.9033 & 0.5906 & 0.0034 & 0.4060 & 0.8879 & 0.0703 & 0.0418 \\
\hline \multirow{5}{*}{ m-2HEAB } & 0.3602 & 0.1562 & 0.4836 & 0.5715 & 0.2273 & 0.2012 & 0.6560 & 0.2557 & 0.0883 \\
\hline & 0.2794 & 0.1120 & 0.6086 & 0.5713 & 0.2021 & 0.2267 & 0.6995 & 0.2416 & 0.0588 \\
\hline & 0.1716 & 0.0688 & 0.7596 & 0.5715 & 0.1715 & 0.2570 & 0.7479 & 0.2167 & 0.0353 \\
\hline & 0.1183 & 0.0484 & 0.8333 & 0.5706 & 0.1434 & 0.2860 & 0.7889 & 0.1892 & 0.0218 \\
\hline & 0.1275 & 0.0523 & 0.8202 & 0.5706 & 0.1290 & 0.3005 & 0.8130 & 0.1709 & 0.0160 \\
\hline \multirow{3}{*}{ m-2HEAP } & 0.1194 & 0.0420 & 0.8386 & 0.5713 & 0.0821 & 0.3467 & 0.7795 & 0.1316 & 0.0888 \\
\hline & 0.0916 & 0.0088 & 0.8996 & 0.5815 & 0.0515 & 0.3670 & 0.8379 & 0.0967 & 0.0653 \\
\hline & 0.0783 & 0.0084 & 0.9133 & 0.5906 & 0.0034 & 0.4060 & 0.8889 & 0.0592 & 0.0518 \\
\hline \multicolumn{10}{|c|}{ 333.15K } \\
\hline IL & $w_{1}^{T}$ & $w_{2}^{T}$ & $w_{3}^{T}$ & $w_{1}^{M}$ & $w_{2}^{M}$ & $w_{3}^{M}$ & $w_{1}^{B}$ & $w_{2}^{B}$ & $w_{3}^{B}$ \\
\hline \multirow{3}{*}{ m-2HEAPr } & 0.2043 & 0.0872 & 0.7085 & 0.4050 & 0.1882 & 0.4068 & 0.6990 & 0.2386 & 0.0624 \\
\hline & 0.1607 & 0.0194 & 0.8199 & 0.4545 & 0.1033 & 0.4422 & 0.8212 & 0.1253 & 0.0535 \\
\hline & 0.1559 & 0.0285 & 0.8157 & 0.4741 & 0.0501 & 0.4758 & 0.8501 & 0.1124 & 0.0376 \\
\hline \multirow{3}{*}{ m-2HEAB } & 0.3203 & 0.0742 & 0.6054 & 0.6195 & 0.1876 & 0.1929 & 0.6993 & 0.2178 & 0.0829 \\
\hline & 0.2429 & 0.0184 & 0.7387 & 0.6220 & 0.1517 & 0.2262 & 0.7510 & 0.1971 & 0.0520 \\
\hline & 0.2242 & 0.0047 & 0.7711 & 0.6173 & 0.1239 & 0.2587 & 0.7886 & 0.1758 & 0.0356 \\
\hline \multirow{4}{*}{$\mathrm{m}-2 \mathrm{HEAP}$} & 0.2092 & 0.0895 & 0.7013 & 0.6015 & 0.1473 & 0,2512 & 0.7460 & 0.1657 & 0.0883 \\
\hline & 0.1994 & 0.0620 & 0.7386 & 0.5813 & 0.0821 & 0.3367 & 0.7795 & 0.1316 & 0.0888 \\
\hline & 0.1816 & 0.0288 & 0.7896 & 0.5915 & 0.0415 & 0.3670 & 0.8379 & 0.0967 & 0.0653 \\
\hline & 0.1683 & 0.0084 & 0.8233 & 0.5978 & 0.0062 & 0.3960 & 0.8889 & 0.0592 & 0.0518 \\
\hline
\end{tabular}


Table 10: Hand Coefficients $\mathrm{k}_{\mathrm{H}}$ and const ${ }_{\mathrm{H}}$ and Othmer-Tobias Coefficientes $\mathrm{k}_{\mathrm{OT}}$ and const ${ }_{\mathrm{OT}}$ of water $+\mathrm{IL}+$ butanol, and respective Correlation Coefficient $\left(\mathrm{R}^{2}\right)$

\begin{tabular}{|c|c|c|c|c|c|c|}
\hline \multicolumn{7}{|c|}{$293.15 \mathrm{~K}$} \\
\hline \multirow{2}{*}{ Systems } & \multicolumn{3}{|c|}{ Hand Test } & \multicolumn{3}{|c|}{ Othmer-Tobias Test } \\
\hline & $\mathrm{k}_{\mathrm{H}}$ & const $_{H}$ & $\mathrm{R}^{2}$ & $\mathrm{k}_{\mathrm{OT}}$ & const $_{\mathrm{OT}}$ & $\mathrm{R}^{2}$ \\
\hline Water + m-2HEAPr + 1-Butanol & 0.1137 & -1.2560 & 0.9408 & 0.8724 & -1.0998 & 0.9924 \\
\hline Water + m-2HEAB + 1-Butanol & 0.1180 & -1.6776 & 0.9857 & 0.6532 & -1.5134 & 0.8090 \\
\hline Water + m-2HEAP +1 -Butanol & 0.3970 & -1.6562 & 0.9519 & 0.5992 & -1.5891 & 0.8588 \\
\hline \multicolumn{7}{|c|}{$313.15 \mathrm{~K}$} \\
\hline Water + m-2HEAPr +1 -Butanol & 0.7841 & -0.3368 & 0.9863 & 0.5901 & -1.002 & 0.9905 \\
\hline Water + m-2HEAB + 1-Butanol & 0.1792 & -1.5701 & 0.9986 & 0.5693 & -1.5113 & 0.9317 \\
\hline Water + m-2HEAP + 1-Butanol & 0.3504 & -1.6529 & 0.9786 & 0.5194 & -1.5819 & 0.9717 \\
\hline \multicolumn{7}{|c|}{$333.15 \mathrm{~K}$} \\
\hline Water + m-2HEAPr +1 -Butanol & 1.0180 & -0.5597 & 0,9319 & 1.0991 & -1.2742 & 0.9910 \\
\hline Water + m-2HEAB + 1-Butanol & 0.3302 & -1.5693 & 0.9490 & 0.6706 & -1.4568 & 0.9977 \\
\hline Water + m-2HEAP +1 -Butanol & 1.5018 & 1.2683 & 0.9586 & 1.6525 & -2.0691 & 0.9923 \\
\hline
\end{tabular}

Table 11: Hand Coefficients $\mathrm{k}_{\mathrm{H}}$ and const ${ }_{\mathrm{H}}$ and Othmer-Tobias Coefficientes $\mathrm{k}_{\mathrm{OT}}$ and const $\mathrm{OT}_{\mathrm{OT}}$ of water $+\mathrm{IL}+$ pentanol, and respective Correlation Coefficient $\left(\mathrm{R}^{2}\right)$

\begin{tabular}{|c|c|c|c|c|c|c|}
\hline \multicolumn{7}{|c|}{$293.15 \mathrm{~K}$} \\
\hline \multirow{2}{*}{ Systems } & \multicolumn{3}{|c|}{ Hand Test } & \multicolumn{3}{|c|}{ Othmer-Tobias Test } \\
\hline & $\mathrm{k}_{\mathrm{H}}$ & const $_{\mathrm{H}}$ & $\mathrm{R}^{2}$ & $\mathrm{k}_{\mathrm{OT}}$ & const $_{\mathrm{OT}}$ & $\mathrm{R}^{2}$ \\
\hline Water + m-2HEAPr + 1-Pentanol & 0.8809 & -0.5011 & 0.9804 & 1.2979 & -1.6252 & 0.9956 \\
\hline Water + m-2HEAB +1 -Pentanol & 0.2704 & -1.1190 & 0.9911 & 0.5972 & -1.3559 & 0.9681 \\
\hline Water + m-2HEAP +1 -Pentanol & 0.1146 & -1.5754 & 0.7818 & 0.2536 & -1.4538 & 0.9624 \\
\hline \multicolumn{7}{|c|}{$313.15 \mathrm{~K}$} \\
\hline Water + m-2HEAPr + 1-Pentanol & 1.2432 & 0.6881 & 0.8973 & 0.8715 & -1.3469 & 0.9820 \\
\hline Water + m-2HEAB +1 -Pentanol & 0.3275 & -1.1029 & 0.9002 & 0.5260 & -1.4513 & 0.9455 \\
\hline Water + m-2HEAP +1 -Pentanol & 0.4937 & -1.0624 & 0.5808 & -1.5397 & -1.1233 & 0.9684 \\
\hline \multicolumn{7}{|c|}{$333.15 \mathrm{~K}$} \\
\hline Water + m-2HEAPr + 1-Pentanol & 0.5539 & -0.7752 & 0.8711 & 1.7485 & -1.5239 & 0.9460 \\
\hline Water + m-2HEAB +1 -Pentanol & 0.1112 & -1.3319 & 0.9996 & 0.8116 & -1.5695 & 0.9352 \\
\hline Water + m-2HEAP +1 -Pentanol & 0.4663 & -1.1068 & 0.9949 & 2.2115 & -1.7117 & 0.9783 \\
\hline
\end{tabular}

Parameters for the UNIFAC model are listed in Tables 12 to 14. UNIFAC tie-lines for m-2HEAB in water-butanol and water-pentanol systems are shown in Figures 7-12. First, fits were made for each alcohol separately as an initial estimate for overall data fitting. This leads to unreal ionic liquid interaction parameters with other groups because different sets are obtained for the same ionic liquid, but is an interesting approach to obtain a initial estimate for generating the final parameters. However, a poor description was obtained for pentanol-water systems, as can be observed in Figures 10-12, and it was useless to try to fit a unique set of parameters because the result would be even worse. A better way of modelling these systems in a group contribution manner is a challenge for future works. Thus, liquid-liquid equilibria of the ionic liquid are well described by UNIFAC for water-butanol, but modelling for the water-pentanol system was not satisfactory.

Table 12: Volume $(\mathrm{R})$ and area $(\mathrm{Q})$ parameters for the UNIFAC model

\begin{tabular}{ccc}
\hline Group & $\mathbf{R}$ & $\mathbf{Q}$ \\
\hline $\mathrm{CH}_{2}{ }^{*}$ & 0.6744 & 0.5400 \\
$\mathrm{CH}_{3}{ }^{*}$ & 0.9011 & 0.8480 \\
$\mathrm{H}_{2}{ }^{*}$ & 0.9200 & 1.4000 \\
$\mathrm{OH}{ }^{*}$ & 1.0000 & 1.2000 \\
m2HEAPr & 7.00 & 5.54 \\
m2HEAB & 7.65 & 5.51 \\
m2HEAP & 8.83 & 6.19 \\
\hline
\end{tabular}

* Values from Magnussen et al. (1981). 
Table 13: UNIFAC Interaction parameters fitted for the systems with butanol

\begin{tabular}{cccc}
\hline & $\begin{array}{c}\mathrm{CH}_{\mathrm{n}}(\mathrm{i})- \\
\mathrm{m} 2 \mathrm{HEAPr}_{(j)}\end{array}$ & $\begin{array}{c}\mathrm{H}_{2} \mathrm{O}(\mathrm{i})- \\
\text { m2HEAPr(j) }\end{array}$ & $\begin{array}{c}\mathrm{OH}(\mathrm{i})- \\
\text { m2HEAPr(j) }\end{array}$ \\
\hline $\mathrm{a}_{\mathrm{ij}}$ & 13.35 & 29.694 & 433.75 \\
$\mathrm{a}_{\mathrm{ji}}$ & 2750.5 & -114.93 & -60.479 \\
\hline & $\mathrm{CH}_{\mathrm{n}}(\mathrm{i})-$ & $\mathrm{H}_{2} \mathrm{O}(\mathrm{i})-$ & $\mathrm{OH}(\mathrm{i})-$ \\
& $\left.\mathrm{m} 2 \mathrm{HEAP}^{\mathrm{j}}\right)$ & $\mathrm{m} 2 \mathrm{HEAP}(\mathrm{j})$ & $\mathrm{m} 2 \mathrm{HEAP}(\mathrm{j})$ \\
\hline $\mathrm{a}_{\mathrm{ij}}$ & 2766.6 & 920.22 & 377.75 \\
$\mathrm{a}_{\mathrm{ij}}$ & 107.99 & -343.52 & 684.64 \\
\hline & $\mathrm{CH}_{\mathrm{n}}(\mathrm{i})-$ & $\mathrm{H}_{2} \mathrm{O}(\mathrm{i})-$ & $\mathrm{OH}(\mathrm{i})-$ \\
& $\mathrm{m} 2 \mathrm{HEAB} \mathrm{j})$ & $\mathrm{m} 2 \mathrm{HEAB}(\mathrm{j})$ & $\mathrm{m} 2 \mathrm{HEAB}(\mathrm{j})$ \\
\hline $\mathrm{a}_{\mathrm{ij}}$ & 367.52 & 357.86 & 1142.90 \\
$\mathrm{a}_{\mathrm{ji}}$ & 110.37 & -192.07 & 1740.60 \\
\hline
\end{tabular}

Table 14: UNIFAC Interaction parameters fitted for the systems with pentanol

\begin{tabular}{cccc}
\hline & $\begin{array}{c}\mathrm{CH}_{\mathrm{n}}(\mathrm{i})- \\
\text { m2HEAPr(j) }\end{array}$ & $\begin{array}{c}\mathrm{H}_{2} \mathrm{O}(\mathrm{i})- \\
\text { m2HEAPr(j) }\end{array}$ & $\begin{array}{c}\text { OH(i)- } \\
\text { m2HEAPr(j) }\end{array}$ \\
\hline $\mathrm{a}_{\mathrm{ij}}$ & 3000.0 & 329.22 & 796.27 \\
$\mathrm{a}_{\mathrm{ji}}$ & -224.48 & -182.39 & 2994.7 \\
\hline & $\mathrm{CH}_{\mathrm{n}}(\mathrm{i})-$ & $\mathrm{H}_{2} \mathrm{O}(\mathrm{i})-$ & $\mathrm{OH}(\mathrm{i})-$ \\
& $\left.\mathrm{m} 2 \mathrm{HEAP}^{\mathrm{j}}\right)$ & $\mathrm{m}^{\mathrm{H}} \mathrm{HEAP}(\mathrm{j})$ & $\mathrm{m} 2 \mathrm{HEAP}(\mathrm{j})$ \\
\hline $\mathrm{a}_{\mathrm{ij}}$ & 2745.5 & -648.14 & 513.12 \\
$\mathrm{a}_{\mathrm{ji}}$ & 84.858 & -205.11 & -69.247 \\
& $\mathrm{CH}(\mathrm{i})-$ & $\mathrm{H}_{2} \mathrm{O}(\mathrm{i})-$ & $\mathrm{OH}(\mathrm{i})-$ \\
& $\mathrm{m} 2 \mathrm{HEAB} \mathrm{j})$ & $\mathrm{m} 2 \mathrm{HEAB}(\mathrm{j})$ & $\mathrm{m} 2 \mathrm{HEAB}(\mathrm{j})$ \\
\hline $\mathrm{a}_{\mathrm{ij}}$ & 205.27 & -345.74 & 2554.4 \\
$\mathrm{a}_{\mathrm{ji}}$ & 81.573 & 776.74 & 375.12 \\
\hline
\end{tabular}

\section{CONCLUSIONS}

Liquid-liquid data for water + ionic liquid + alcohol were studied at $\mathrm{P}=101.2 \mathrm{kPa}$ and $293.15,313.15$ and $333.15 \mathrm{~K}$. The alcohols used were butanol and pentanol and the ionic liquids are three protic N-methyl2-hydroxyethylammonium alkylates (propionate, butanoate and pentanoate). It was found that, in all conditions evaluated, the IL showed a high miscibility in both solvents, and induced a salting in effect in the systems. Increasing the temperature, the miscibility gap is enhanced. The surfactant characteristics of the ionic liquid increase as the anion chain is increased and the mutual solubility of water-alcohol are augmented. Data were considered to be consistent. UNIFAC was used to correlate liquid-liquid equilibrium behavior for water-alcohol systems. However, using fitting parameters for each alcohol separately led to unreal and different parameters for the same ionic liquid. The systems with water-pentanol were not satisfactorily described by the UNIFAC method. A better way of modelling these data is a challenge for future work. The results confirm the high hydrophilicity of these ionic liquids, as their butanol-water and pentanol-water partition coefficients are normally lower than 1 .

\section{ACKNOWLEGMENTS}

The authors are grateful to CAPES/Brazil for M.Sc. grants for F. Ganem and K. Amorim. Financial support from $\mathrm{CNPq} /$ Brazil is also acknowleged (Grant 306560/2013-5, project 475883/2012-8 and E. Souza MSc. Grant). The authors are also grateful to FAPESB/ SECTI for A. Soares' undergraduate grant.

\section{REFERENCES}

Alvarez, V.H., Mattedi, S., Aznar, M., Density, Refraction Index and Vapor-Liquid Equilibria of N-Methyl-2-Hydroxyethylammonium Butyrate plus (Methyl Acetate or Ethyl Acetate or Propyl Acetate) at Several Temperatures. J. of Chem. Thermodyn., 62, 130-141 (2013).

Alvarez V.H., Dosil N., Gonzalez-Cabaleiro R., Mattedi S., Martin-Pastor M., Iglesias M., Navaza J.M., Brønsted Ionic Liquids for Sustainable Processes: Synthesis and Physical Properties. J. Chem. Eng. Data, 55, 625-632 (2010).

Carvalho, P.J., Coutinho, J.A.P., The polarity effect upon the methane solubility in ionic liquids: a contribution for the design of ionic liquids for enhanced $\mathrm{CO} 2 / \mathrm{CH} 4$ and $\mathrm{H} 2 \mathrm{~S} / \mathrm{CH} 4$ selectivities, Energy Environ. Sci., 4, 4614 (2011).

Greaves, T.L.; Drummond, C.J., Solvent nanostructure, the solvophobic effect and amphiphile selfassembly in ionic liquids. Chem. Soc. Rev., 42, 1096-1120, 2013.

Hand, D. B., Dineric distribution, J. Phys. Chem. 34, 1961-2000 (1930).

Latala, A., Stepnowski P, Nedzi M., Mrozik W., Marine Toxicity Assessment of Imidazolium Ionic Liquids: Acute Effects on the Baltic Algae Oocystis submarina and Cyclotella meneghiniana. Aquatic Toxicolog., 73(1) 91-98 (2005).

Li, Y., Figueiredo, E.J.P., Santos, M., Santos, J., Talavera-Prieto, N.M.C., Carvalho, P.J., Ferreira, A.G.M., Mattedi, S., Volumetric and acoustical properties of aqueous mixtures of $\mathrm{N}$-methyl2-hydroxyethylammonium propionate at $\mathrm{T}=$ (298.15 K to 333.15) K. Journal of Chemical Thermodynamics, 88, 44-60 (2015). 
Li, Y., Figueiredo, E.J.P., Santos, M., Santos, J., Talavera-Prieto, N.M.C., Carvalho, P.J., Ferreira, A.G.M., Mattedi, S., Volumetric and acoustical properties of aqueous mixtures of N-methyl-2hydroxyethylammonium butyrate and $\mathrm{N}$-methyl-2hydroxyethylammonium pentanoate at $\mathrm{T}=(298.15$ to 333.15)K., J. of Chem. Thermodyn., 97, 191205 (2016).

Kennedy, D.F., Drummond C.J., Large Aggregated Ions Found in Some Protic Ionic Liquids. J. Phys. Chem., B, 113(17), 5690-5693 (2009).

Magnussen, T.; Rasmussen, P.; Fredenslund, A., UNIFAC Parameter Table for Prediction of LiquidLiquid Equilibria. Ind. Eng. Chem. Process Des. Dev., 20(2), (331-339 (1981).

Merchuk J.C., Andrews B. A., Asenjo J.A., Aqueous two-phase systems for protein separation. Studies on phase inversion, J Chromatogr. B Biomed. Sci. Appl. 711, 285-293 (1998).

Nann, A.; Held, C.; Sadowski, G., Liquid-Liquid Equilibria of 1-Butanol/Water/IL Systems. Ind. Eng. Chem. Res., 52, 18472-18481 (2013).

Nemati-Kande, E., Shekaari, H., Zafarini-Moattar, M.T., Binodal Curves and Tie-Lines of Aliphatic Alcohols plus Diammonium Hydrogen Citrate plus Water Ternary Systems: Measurement and Modeling, J. Chem. Eng., Data 57, 1678-1688 (2012).

Oliveira, L. H. de; Álvarez, V. H., Aznar, M., Liquid Liquid Equilibrium in N-Methyl-2hydroxyethylammonium Acetate, Butanoate, or Hexanoate Ionic Liquids + Dibenzothiophene + n-Dodecane Systems at 298.2 K and Atmospheric Pressure. J. of Chem. \& Eng. Data, 57, 744-750 (2012).

Oliveira, M.V.S., Vidal, B.T., Melo, C.M., Miranda, R.C.M., Soares, C.M.F.; Coutinho, J.A.P., Ventura, S.P.M., Mattedi, S., Lima, A.S., (Eco)toxicity and biodegradability of protic ionic liquids. Chemosphere (Oxford), 147, 460-466 (2016).
Oliveira, M.V., Ros, P.C.M., Castro, H.F., Mattedi, S., Soares, C.M.F. , Lima, A.S., Transesterification of babassu oil catalyzed by Burkholderia cepacia encapsulated in sol-gel matrix employing protic ionic liquid as an additive. Acta Scientiarum. Technology, 36, 445-451 (2014).

Othmer, D.F., Tobias, P. E., Tie-line Correlation. Industrial and Engineering Chemistry, 34, 693-696 (1942).

Peric, B., Sierra, J., Martí, E., Cruañas, R., Garau, M. A., A comparative study of the terrestrial ecotoxicity of selected protic and aprotic ionic liquids, Chemosphere, 108, 418-425 (2014).

Santos, G.R., UNIFAC-CAMPINAS: Um novo modelo UNIFAC para a predição do equilíbrio líquido-líquido. DSc. Thesis. Chemical Engineering Faculty - UNICAMP. 2005. Campinas, São Paulo.

Santos, D., Costa, F., Franceschi, E., Santos, A., Dariva, C., Mattedi, S., Synthesis and physicochemical properties of two protic ionic liquids based on stearate anion. Fluid Phase Equilibria, 376, 132-140 (2014).

Souza, R.L., Faria, E.L.P., Figueiredo, R.T., Freitas, L.S., Iglesias, M., Mattedi, S., Zanin, G.M., Santos, O.A.A., Coutinho, J.A.P. ; Lima, A.S., Soares, C.M.F., Protic ionic liquid as additive on lipase immobilization using silica sol gel. Enzyme and Microbial Technology , 52, 141-150 (2013).

Stragevitch, L., d'Ávila, S.G., Application of a generalized maximum likelihood method in the reduction of multicomponent liquid-liquid equilibrium data. Braz. J. Chem. Eng., 14, 41-52 (1997)

Talavera-Prieto, N.M.C., Ferreira, A.G.M., Simões, P.N., Carvalho, P., Mattedi, S., Coutinho, J.A.P., Thermophysical characterization of N-methyl-2 -hydroxyethylammonium carboxilate ionic liquids, J. of Chem. Thermodyn., 68, 221-234 (2014). 